\title{
Treatment success rates among drug susceptible tuberculosis patients notified from the private sector in West Bengal, India
}

\author{
Vivek Sharma ${ }^{1}$, Pruthu Thekkur ${ }^{2,3}$, Poonam R. Naik ${ }^{4}$, Brojo Kishore Saha ${ }^{5}$, Niraj Agrawal ${ }^{6}$, \\ Milan K. Dinda ${ }^{7}$, S. Parthiban ${ }^{7}$, Abhijit Dey ${ }^{7}$, Basavaraj Poojar ${ }^{8}$, Amrita N. Shamanewadi', \\ Srinath Satyanarayana ${ }^{2,3}$
}

${ }^{1}$ John Snow India (JSI), Tuberculosis Health Action and Learning Initiative (THALI), West Bengal, India; ${ }^{2}$ Centre for Operational Research, International Union Against Tuberculosis and Lung Disease, Paris, France; ${ }^{3}$ The Union South East Asia Office (The USEA), New Delhi, India; ${ }^{4}$ Department of Community Medicine, Yenepoya

Correspondence: Vivek Sharma, Monitoring \& Evaluation Advisor, JSI India, A-201, Vrundalaya Greens, Atladara, Nr. Cosmos Commercial Building, Padra Road, Baroda, 390012 Gujarat, India. Tel.+91.9835352784. E-mail: vivekiips@hotmail.com

Author's contributions: VS, Principal Investigator and corresponding author, conceived the study/design of the protocol, acquisition of data, data analysis/interpretation, drafting/critically reviewing the paper, giving approval for the final version to be published; PT, PN, conception/design of the protocol, data analysis/interpretation, critically reviewing the paper, giving approval for the final version to be published; BKS, MKD, $\mathrm{AD}$, acquisition of data, critically reviewing the paper, giving approval for the final version to be published; SP, BP, ANS, acquisition of data, critically reviewing the paper, giving approval for the final version to be published; SS, Role of mentor/senior investigator during conception, design, acquisition of data, critically reviewing the paper, giving approval for the final version to be published.

Conflict of interest: The authors declare that they have no competing interests, and all authors confirm accuracy.

Funding: No fund is received from any sources or donors.

Ethics approval: The study was approved by the Ethics Advisory Group of the International Union Against Tuberculosis and Lung Disease, Paris, France. Formal written permission was taken from the State TB officer, where the study had been conducted.

Acknowledgments: This research was conducted through the Structured Operational Research and Training Initiative (SORT IT), a global partnership led by the Special Program for Research and Training in Tropical Diseases at the World Health Organization (WHO/TDR). The model is based on a course developed jointly by the International Union Against Tuberculosis and Lung Disease (The Union) and Medécins sans Frontières (MSF/Doctors Without Borders). The specific SORT IT program which resulted in this publication was jointly developed and implemented by the Union South-East Asia Office, New Delhi, India; the Centre for Operational Research, The Union, Paris, France; the Operational Research Unit (LUXOR), MSF Brussels Operational Centre, Luxembourg, USAID New Delhi.

We extend our thanks to Mr. Dibyendu Chatterjee for the necessary help and support for the study.

Key words: Operational research; private sector; SORT IT; treatment outcome; tuberculosis.

Received for publication: 9 October 2020.

Accepted for publication: 11 December 2020.

${ }^{\circ}$ Copyright: the Author(s), 2021

Licensee PAGEPress, Italy

Monaldi Archives for Chest Disease 2021; 91:1555

doi: 10.4081/monaldi.2021.1555

This article is distributed under the terms of the Creative Commons Attribution Noncommercial License (by-nc 4.0) which permits any noncommercial use, distribution, and reproduction in any medium, provided the original author(s) and source are credited. Medical College, Yenepoya (Deemed to be University), Mangalore, India; ${ }^{5}$ State $T B$ Office, Department of Health and Family Welfare, West Bengal, India; ${ }^{6} J o h n$ Snow India (JSI), Tuberculosis Health Action and Learning Initiative (THALI), New Delhi, India; ${ }^{7}$ World Health Partners, Tuberculosis Health Action and Learning Initiative (THALI), West Bengal, India; ${ }^{8}$ Department of Pharmacology, Kasturba Medical College, Mangalore, India; ${ }^{9}$ Department of Community Medicine, MVJ Medical College and Research Hospital (MVJMCRH), Dandupalya, Hoskote, Bengaluru rural, India

\section{Abstract}

In India, an estimated $50 \%$ of tuberculosis (TB) patients are diagnosed and managed by private providers (PPs). However, there is limited information on treatment outcomes among those treated by PPs largely because of a lack of systems for patient follow-up and documentation of the outcomes. Tuberculosis Health Action Learning Initiative (THALI) project implemented in six districts of West Bengal, supported PPs in managing TB patients and systematically documented the TB treatment outcomes of such patients. This provided us an opportunity to describe the treatment outcomes and assess factors associated with 'unsuccessful outcomes' among TB patients notified by PPs from January to April 2018. This was a retrospective cohort study using routinely collected data. During the study reference period, 2347 patients were notified from 389 PPs. The patient's mean (SD) age was 39.9 (17.2) years and 61\% were males. Of the total, about $86 \%$ had pulmonary TB, $95 \%$ were new cases, and $23 \%$ were microbiologically confirmed (either on sputum smear microscopy or Xpert assay). Among 2347 patients, 19\% received free drugs from the National Tuberculosis Program (NTP) under the supervision of PPs while the rest purchased anti-TB drugs from the private pharmacies. The 'successful outcomes' (cured and treatment completed) were seen in $86.1 \%$ (95\% confidence interval (CI), 84.6\%-87.4\%) patients and $8.6 \%$ were 'not evaluated'. The patients who received free NTP drugs (adjusted relative risk-4.0 (95\% CI: 3.1-5.0)) had a higher risk of 'unsuccessful outcomes' than those who availed of drugs from private pharmacies. The high 'successful outcomes' among TB patients treated by PPs are promising. However, higher 'unsuccessful outcomes' among patients on free NTP drugs need to be explored, and suitable corrective actions have to be taken. 


\section{Introduction}

With an estimated 2.7 million new tuberculosis (TB) patients in the year 2018, India contributed to more than $25 \%$ of the estimated incident cases globally [1]. The National Tuberculosis Programme (NTP) of India has made steady progress in providing quality-assured TB diagnostic and treatment services in the public sector [2]. Despite this, an estimated $50 \%$ of the TB patients present initially to private providers (PPs) and eventually managed within the private sector $[3,4]$.

The previous studies have reported that TB patients in the private sector are exposed to suboptimal diagnostic and treatment regimens $[5,6]$. Due to deficiencies in treatment adherence monitoring and patient follow-up mechanism, the TB treatment outcomes of patients managed in the private sector are rarely ascertained and reported to NTP $[7,8]$. Thus, there is limited information on the TB treatment outcomes of patients managed in the private sector. Even the research studies on TB treatment outcomes in the private sector are mostly limited to single tertiary care hospitals and have reported a low rate of 'successful outcomes' (cured and treatment completed) [9-11].

'Tuberculosis Health Action Learning Initiative' (THALI)' project was implemented in six urban districts of West Bengal by the consortium of non-governmental organisations (NGOs). The THALI project supported formal PPs (medical doctors with minimum qualification of Bachelor of Medicine, Bachelor of Surgery; MBBS) for providing optimal TB care at their clinics or hospitals. All formal PPs in the project districts were mapped and the project staff engaged the PPs willing to be part of the project. The field officers (FOs) of the project assisted enrolled PPs in notifying the diagnosed TB patients to NTP, provided adherence support to TB patients by making house visits and a weekly call through the 'callcentre' and tracked the patients till treatment outcome is ascertained. The project also supported PPs in procuring free NTP anti-TB drugs for managing patients who could not afford the anti-TB drugs in private pharmacies. The THALI project used a web-based information communication technology (ICT) system to report and record details of TB treatment service delivery and patient's treatment outcomes. The project incentivized PPs with 500 Indian rupees (INR) per treated TB patient for ascertaining the TB treatment outcomes and the FOs verified the reported treatment outcomes. Thus, the systematically documented treatment outcomes of a large cohort of TB patients supported under the project provided an opportunity to fill information gap on the private sector's treatment outcomes. Hence, we aimed to describe the treatment outcomes and assess the factors associated with 'unsuccessful outcomes' among drug-sensitive TB patients notified and initiated on treatment by the PPs in the six selected districts of West Bengal from January to April 2018.

\section{Methods}

\section{Study design}

This was a retrospective cohort study using secondary data collected routinely by the THALI project for programme monitoring and reporting to the NTP.

\section{Study setting}

\section{General setting}

The West Bengal state is situated in the Eastern part of India, between the Himalayas and the Bay of Bengal. During the 2011 census, the state had about 91 million population with a density of 1030 individuals per square kilometre. In 2017, around 104 thousand incident TB patients were notified to NTP in West Bengal, of which around $15 \%$ were from the private sector.

\section{Specific setting}

THALI is a USAID-funded project implemented by a consortium of three NGOs in Kolkata, Howrah, Hoogly, North-24Parganas, South-24-Parganas, and East Medinipur districts of West Bengal.

\section{Engagement of PPs}

In 2017, all the standalone clinics, hospitals, and nursing homes with the formal PPs qualified with Bachelor in Medicine and Bachelor in Surgery (MBBS) or above in project districts were mapped. Of the total 1404 formal PPs mapped, 1050 (75\%) signed a memorandum of understanding (MoU) and enrolled in the THALI project. The project had twenty-three FOs, who engaged with pre-assigned PPs. The PPs were trained on NTP guidelines for diagnosing, managing and ascertaining of treatment outcomes of TB patients. A ‘call-centre' was developed to provide ICT support. The PPs could reach out to this call-centre from their registered mobile numbers during working hours to support notification, diagnosis, and treatment of TB patients.

\section{Diagnosis, notification and treatment by THALI supported PPS}

The PPs contacted the 'call-centre' and reported details of the presumptive TB patient (PTBP) detected in the health facility. The 'call-centre' alerted the respective FOs to facilitate provider and PTBP to complete the diagnostic algorithm in line with NTP guidelines. The Xpert MTB/Rif assay at a public health facility was made available free of cost for the diagnosis of all the PTBPs detected by PPs. The PTBPs with Mycobacterium tuberculosis bacilli detected either phenotypically (sputum smear microscopy or culture) or genotypically (Xpert assay) are considered 'microbiologically confirmed TB'. Those individuals with high suspicion of TB among PPs either based on clinical examination or through non-TB specific diagnostic tools like chest $\mathrm{x}$-ray are diagnosed as 'clinically diagnosed TB'. The TB patients were classified as per NTP guidelines and the PPs informed the patient details to THALI call-centre.

The FOs supported the notification of the TB patient to the NTP. FOs contacted the patient at either the health facility or the patient's residence. The patient was registered in the 'THALI patient database' maintained at the call-centre and patient details were periodically updated. The patient was informed regarding the adherence support provided through the THALI project.

In consultation with treating PPs, the TB patients who were unable to purchase anti-TB drugs in private pharmacies were offered free drugs provided by NTP. The patient continued consulting the PP but received free anti-drugs provided by the NTP. The FOs procured NTP anti-drugs and made it available for the patient. The patients could contact the call-centre to get information related to TB and treatment. The patient used to get an adherence support call from the "call-centre" once a week. The FO made at least three patient house visits during treatment; soon after notification, end of the intensive phase (IP) and the continuation phase (CP). In case the patient was not taking the treatment or not visited the PP as prescribed, additional visits were made to track the patients. The house visits were made only when the patient was residing in the THALI project districts. During the final house visit, FOs verified the patient's treatment card and collected details on completion of treatment and end of follow-up test results. After the patient's 
house's final visit, the FOs visited the PP, verified the TB register, and discussed the doctor's treatment outcome. After physical verification of the data and discussion, the doctor made the final call to the call-centre to declare the treatment outcome. The call-centre contacted the patients on the declaration of treatment outcome and interviewed them to validate the declared treatment outcomes (Supplementary Figure 1). The programmatic TB treatment outcomes were ascertained as per the NTP guidelines of India (Supplementary Table1). The PPs were incentivised for adhering to project requirements while managing the TB patients. The PPs were offered 500 INR against three agreed deliverables while managing each patient. The first deliverable was to identify and notify the TB patient yo NTP. The second was to record the patient's status at the end of intensive phase of TB treatment. The third was to report the TB treatment outcome of the patient.

\section{Recording and reporting}

The socio-demographic and clinical details of the notified TB patients were recorded by the health assistant or the PPs in the paper-based patient registers maintained at each of the private health facilities either. The FOs supported the health assistants or the PPs in maintaining the patient registers and checked for any missing information or inconsistency in recording. The FOs informed the patient details recorded in the patient register, which were digitised at the THALI project's call-centre. The FOs documented the treatment and follow-up details in the mobile application during their patient home visit. The information captured in the mobile application was used to update the patient register maintained at the health facilities and the electronic database managed at the call-centre. The PPs recorded TB treatment outcomes in the patient register and the FOs documented the treatment outcomes in the mobile application after validation through house visit. The data in the mobile application was shared with the callcentre to update the database.

\section{Study population}

All the TB patients notified from January to April 2018 by PPs engaged with the THALI project in six urban districts of West Bengal were included in the study. The drug-resistant TB (23 during the study period) patients were excluded from the study as PPs referred these patients to public health facilities for further management and also, the THALI project did not conduct follow-up of these patients.

\section{Data variables, sources of data, and data collection}

Data on socio-demographic, clinical, and treatment characteristics like THALI case ID, age, gender, district of patient, state of patient, district of provider, type of TB (microbiologically confirmed or clinically diagnosed), site (pulmonary or extra-pulmonary), previous history of TB treatment, human immunodeficiency virus (HIV) status, diabetes mellitus status, type of health facility, education of provider, source of ant-TB drugs, programmatic TB treatment outcomes and date of TB treatment outcomes were extracted from electronic THALI patient database.

\section{Data entry and analysis}

Data extracted from the electronic database in Microsoft Excel was analyzed using Stata version 12.0 (StataCorp LP, College Station, TX, USA). Demographic, clinical, and treatment characteristics were summarized as percentages. TB treatment outcomes were summarized as the proportion with a $95 \%$ confidence interval (CI). TB treatment outcomes were categorized into successful (cured and treatment completed) and unsuccessful (failure, lost to follow up, died, switched to category IV, and not evaluated) outcomes.

The association between socio-demographic and baseline clinical characteristics with 'unsuccessful' treatment outcomes was assessed using bivariate log-binomial regression. To assess the independent association, a cluster (individual PP) adjusted generalized linear model (Poisson regression) with variables having $\mathrm{p}$ value $<0.25$ in the bivariate log-binomial regression was developed. Adjusted relative risk (aRR) with a 95\% CI was calculated as a measure of association.

\section{Result}

In total, 2347 TB patients were notified by 389 PPs during the study reference period. Of the notified, the mean (SD) age was $39.9(17.2)$ years, and $1436(61.2 \%)$ were males. Of the total, about $2028(86 \%)$ of the patients had pulmonary TB, 2225 (95\%) were new cases, and $1820(77 \%)$ were clinically diagnosed. HIV status was unknown for 2037 (87\%) of the TB patients, and diabetes status was unknown for $1526(65 \%)$ of study participants. Of the 2347 notified TB patients, 365 (16\%) visited PPs from a different district than where the health facility is located. The demographic and clinical characteristics of the TB patients are given in Table 1.

Of the 2347 TB patients, 2020 (86.1\%, 95\%CI- 84.6-87.4) had 'successful outcome'. Of the total, $201(8.6 \%)$ were 'not evaluated'. The treatment outcomes of the TB patients stratified with receipt of anti-TB drugs from NTP and private pharmacies are described in Table 2. The reasons for 'not evaluated' were not documented under the project.

The association between patient characteristics and the unsuccessful treatment outcome is shown in Table 3. The patients who received free NTP drugs (aRR-4.0 (95\% CI: 3.1-5.0)) had a higher risk of 'unsuccessful outcomes' than those who availed of drugs from private pharmacies.

\section{Discussion}

This is the first study from India assessing the TB treatment outcomes among a large cohort of drug-susceptible and mainly clinically diagnosed TB patients treated within the private sector. The private sector's treatment outcomes were promising, as $86 \%$ of TB patients had 'successful outcomes'. Despite a well-established mechanism for tracking patients through FOs and ICT support, about $9 \%$ of TB patient's treatment outcomes were 'not evaluated'. Those patients who received anti-TB drugs free of cost from NTP were at higher risk of 'unsuccessful outcomes' than those who availed it from private pharmacies.

In 2016, a study from a private tertiary care hospital in Kerala, India, reported a treatment success rate of $70 \%$ and $75 \%$ among pulmonary and extrapulmonary TB patients, respectively [12]. In 2014, a tertiary hospital of Columbia reported a treatment success rate of $36 \%$, while $37 \%$ of TB patients were not evaluated [13]. The high rate of a successful outcome in the current study might be due to the following reasons. First, most patients (99\%) were treated at standalone clinics and could have the less severe disease than patients seeking care from tertiary hospitals. Second, the TB patients in the current study received patient adherence support through the THALI project, which might have improved the TB treatment outcomes. Third, the free anti-TB drugs from NTP were 
made available through the THALI project, which might have reduced the patient attrition from care due to the inability to pay for drugs at private pharmacies. The THALI project's support with anti-TB drugs from NTP meant PPs need not refer the non-affordable patients to public health facilities for further management. Thus, it limited the potential attrition during such referrals. Fourth,

Table 1. Socio-demographic, clinical characteristics and provider related characteristics of drug-susceptible TB patients registered for treatment with the private providers in selected districts of West Bengal between January and April-2018.

\begin{tabular}{lc} 
Characteristics & Total, $\mathbf{n}(\%)$ \\
Total & $2347(100)$ \\
Age in years & \\
$0-14$ & $81(3.5)$ \\
$15-24$ & $420(17.9)$ \\
$25-34$ & $495(21.1)$ \\
$35-44$ & $431(18.4)$ \\
$45-54$ & $393(16.8)$ \\
$55-64$ & $285(12.1)$ \\
$\geq 65$ & $242(10.3)$ \\
\hline Gender & \\
Male & $1436(61.2)$ \\
Female & $911(38.8)$ \\
Type of TB & \\
Microbiologically confirmed & $527(22.4)$ \\
Clinically diagnosed & $1820(77.6)$ \\
\hline Site of TB & \\
Pulmonary TB & $2028(86.4)$ \\
Extra Pulmonary TB & $319(13.6)$ \\
Category of TB Treatment & \\
New & $2225(94.8)$ \\
Previously treated & $113(4.8)$ \\
TA-LTFU & $2(0.1)$ \\
TAF & $7(0.3)$ \\
\hline
\end{tabular}

\section{HIV status}

Positive

Negative

$4(0.2)$

Unknown

$306(13.0)$

$2037(86.8)$

$\begin{array}{lc}\text { Diabetes status } & 212(9.0) \\ \text { Diabetic } & 609(26.0) \\ \text { Non-diabetic } & 1526(65.0) \\ \text { Unknown }\end{array}$

Type of health facility

$\begin{array}{lc}\text { Clinic } & 2314(98.6) \\ \text { Hospital/Nursing home } & 33(1.4)\end{array}$

\begin{tabular}{lc} 
Education of Provider & \\
MBBS & $883(37.6)$ \\
MBBS with Diploma/MD & $1464(62.4)$ \\
\hline
\end{tabular}

\section{District}

$\begin{array}{lc}\text { Kolkota } & 1090(46.4) \\ \text { Howrah } & 434(18.5) \\ \text { Hooghly } & 216(9.2) \\ \text { North 24 Parganas } & 337(14.4) \\ \text { South 24 Parganas } & 181(7.7) \\ \text { East Medinipur } & 89(3.8)\end{array}$

\begin{tabular}{lc} 
TB care in the district of residence & \\
Same district & $1982(84.4)$ \\
Different district & $365(15.6)$ \\
\hline
\end{tabular}

Anti-TB drugs from

NTP $\quad 444(18.9)$

Private pharmacies 1903 (81.1)

TB, tuberculosis; NTP, National Tuberculosis Programme; TA-LTFU, treatment after loss to follow-up; TAF, treatment after failure; HIV, human immunodeficiency virus; MBBS, Bachelor of Medicine and Bachelor of Surgery; MD,Doctor of Medicine. the treatment outcomes were 'not evaluated' in less than $10 \%$ of the patients than previous studies, which had $20 \%$ to $30 \%$ [14]. These 'not evaluated' were considered as 'unsuccessful outcomes'. Though the proportion with 'not evaluated' was lower in the current study compared to previous studies in the private sector, it was higher than that in the public sector (2\%) in West Bengal [15].

The percentage with 'successful outcomes' among the TB patients in the private sector was on par with the $85 \%$ reported for patients treated under NTP in West Bengal [15]. However, in the current study, TB patients' treatment success rate receiving free anti-TB drugs from NTP in the private sector was relatively low $(63 \%)$. The free NTP drugs were made available to only those who could not afford to purchase the drugs from private pharmacies. The majority of such patients could be from a lower socioeconomic status. We strongly believe that the lower socioeconomic status and its associated challenges than anti-TB drugs from NTP are responsible for low 'successful outcomes' in those receiving free drugs. However, few PPs reported increased adverse drug reactions among patients on fixed-dose combinations (FDC) drugs of NTP and expressed concerns on treatment adherence. There is a need for further research on the potential reason for the low 'successful outcomes' among those on anti-TB drugs from NTP.

The study has a few strengths. First, the study was conducted on a large sample of TB patients treated from about four hundred PPs, which was about $75 \%$ of all PPs in the study districts. This could have enhanced the results' external validity due to variations in patient characteristics and the variation in the TB management protocols adopted by each PP. Second, the standard protocol for ascertaining and validating treatment outcomes limited the potential biases like social desirability bias by relying only on outcomes reported by PPs and the inter-observer bias with multiple FOs involved in ascertaining the 'treatment outcomes'. Third, the data quality was assured as the project's robust data management system with internal checks was used for data capture. Fourth, the Strengthening the Reporting of Observational Studies in Epidemiology (STROBE) guidelines was used to report the study findings.

The study has certain limitations. First, the study was conducted in the THALI project districts and might not completely mimic the management of TB patients by PPs elsewhere. The patient adherence support during the treatment and the incentives to PPs for declaring the 'treatment outcomes' might have led to higher rates of 'successful outcomes' and a lower proportion of 'not evaluated'. Second, about $9 \%$ of treatment outcomes were 'not evaluated' and considered 'unsuccessful' outcomes. However, like most of the 'not evaluated' cases are unaccounted loss to follow-up (LTFU), this might not have grossly underestimated the rate of 'successful outcomes'. Third, the details of various drug regimens prescribed by the PPs were not available in the database. This limited the opportunity to study the pattern of treatment regimens used and the difference in treatment outcomes across the regimens used by the PPs. Fourth, the potential confounders like socioeconomic status, education, adherence during treatment and family support during treatment were not accounted for in the model. This could have reduced the model's validity for assessing factors associated with 'unsuccessful outcomes'. Fifth, the drug-resistant TB patients were not included in the study and their treatment outcomes have not been captured.

The study has a few implications and recommendations. First, there was a high rate of 'successful outcomes' among TB patients managed in the private sector supported by the THALI project. The NTP can adopt models similar to the THALI project to support the management of TB patients in the private sector through dedicated health workers like FOs to engage with PPs for quality TB service provision and track TB patients with adherence support. 
Table 2. TB treatment outcomes of drug-susceptible TB patients registered for treatment with a private provider (stratified by source of anti-TB drugs) in selected districts of West Bengal between January and April-2018.

\begin{tabular}{lccc}
\hline TB treatment outcome & anti-TB drugs & anti-TB drugs & Total, n (\%) \\
\hline & from private pharmacies, $\mathbf{n}(\%)$ & from NIP, n (\%) & $2020(86.1)$ \\
Successful & $1732(91.0)$ & $288(64.9)$ & $28(1.2)$ \\
Cured & $6(0.3)$ & $22(5.0)$ & $1992(84.9)$ \\
Treatment completed & $1726(90.7)$ & $266(59.9)$ & $327(13.9)$ \\
Unsuccessful & $171(9.0)$ & $156(35.1)$ & $1(0.04)$ \\
Failure & $0(0.0)$ & $1(0.23)$ & $88(3.8)$ \\
Lost to follow up & $34(1.8)$ & $10(2.3)$ & $20(0.9)$ \\
Died & $10(0.5)$ & $5(1.1)$ & $17(0.7)$ \\
Shift to Cat IV & $12(0.6)$ & $86(19.4)$ & $201(8.6)$ \\
Not evaluated & $115(6.0)$ & & \\
\hline
\end{tabular}

*Percentage calculated with the total number of individuals in each group as the denominator; TB, tuberculosis; NTP, National Tuberculosis Program; Cat IV, drug regimen used to treat multi-drug resistant TB patients.

Table 3. Association of socio-demographic, clinical and provider-related factors with unsuccessful outcomes among drug-susceptible TB patients registered for treatment with private providers in selected districts of West Bengal from January to April-2018.

\begin{tabular}{|c|c|c|c|c|c|}
\hline Characteristic & Total & $\begin{array}{c}\text { Unsuccessful } \\
\text { outcome, n (\%)* }\end{array}$ & $\begin{array}{l}\text { Unadjusted RR } \\
\qquad(95 \% \mathrm{CI})\end{array}$ & $\begin{array}{l}\text { Adjusted RR } \\
(95 \% \text { CI) }\end{array}$ & p-value \\
\hline Total & 2347 & $327(13.9)$ & & & \\
\hline $\begin{array}{l}\text { Age in years } \\
0-14 \\
15-24 \\
25-34 \\
35-44 \\
45-54 \\
55-64 \\
\geq 65 \\
\end{array}$ & $\begin{array}{c}81 \\
420 \\
495 \\
431 \\
393 \\
285 \\
242\end{array}$ & $\begin{array}{l}13(16.1) \\
44(10.5) \\
64(12.9) \\
77(17.8) \\
57(14.5) \\
33(11.6) \\
39(16.1)\end{array}$ & $\begin{array}{l}1.5(0.9-2.7) \\
1 \\
1.2(0.9-1.8) \\
1.7(1.2-2.4) \\
1.4(1.0-2.0) \\
1.1(0.7-1.7) \\
1.5(1.0-2.3)\end{array}$ & $\begin{array}{c}1.7(1.0-2.7) \\
1 \\
1.6(1.1-2.1) \\
2.2(1.5-3.2) \\
1.7(1.2-2.5) \\
1.4(0.9-2.2) \\
2.1(1.3-3.2)\end{array}$ & $\begin{array}{c}0.038 \\
\\
0.008 \\
<0.001 \\
0.009 \\
0.129 \\
0.002\end{array}$ \\
\hline $\begin{array}{l}\text { Gender } \\
\text { Male } \\
\text { Female }\end{array}$ & $\begin{array}{c}1436 \\
911\end{array}$ & $\begin{array}{l}203(14.1) \\
124(13.6)\end{array}$ & $\begin{array}{c}1.0(0.8-1.3) \\
1\end{array}$ & & \\
\hline $\begin{array}{l}\text { Type of TB } \\
\text { Microbiologically confirmed } \\
\text { Clinically diagnosed }\end{array}$ & $\begin{array}{c}527 \\
1820\end{array}$ & $\begin{array}{c}89(16.9) \\
238(13.1)\end{array}$ & $\begin{array}{c}1.3(1.0-1.6) \\
1\end{array}$ & $\begin{array}{c}1.2(0.9-8.5) \\
1\end{array}$ & 0.164 \\
\hline $\begin{array}{l}\text { Site of TB } \\
\text { Pulmonary TB } \\
\text { Extra pulmonary TB }\end{array}$ & $\begin{array}{l}2028 \\
319\end{array}$ & $\begin{array}{l}280(13.8) \\
47(14.7)\end{array}$ & $1.1(0.8-1.4)$ & & \\
\hline $\begin{array}{l}\text { Category of TB } \\
\text { New } \\
\text { Previously treated }\end{array}$ & $\begin{array}{c}2225 \\
122 \\
\end{array}$ & $\begin{array}{l}303(13.6) \\
24(19.7)\end{array}$ & $\begin{array}{c}1 \\
1.4 \\
(1.0-2.1)\end{array}$ & $\begin{array}{c}1 \\
1.1(0.8-1.6)\end{array}$ & 0.606 \\
\hline $\begin{array}{l}\text { HIV status } \\
\text { Positive } \\
\text { No/unknown }\end{array}$ & $\begin{array}{c}4 \\
2343\end{array}$ & $\begin{array}{c}1(25.0) \\
326(13.9)\end{array}$ & $\begin{array}{c}1.8(0.3-9.8) \\
1\end{array}$ & & \\
\hline $\begin{array}{l}\text { Diabetes status } \\
\text { Diabetic } \\
\text { No/unknown } \\
\end{array}$ & $\begin{array}{c}212 \\
2135\end{array}$ & $\begin{array}{c}32(15.1) \\
295(13.8)\end{array}$ & $\begin{array}{c}1.1(0.8-1.5) \\
1\end{array}$ & & \\
\hline $\begin{array}{l}\text { Type of health facility } \\
\text { Clinic } \\
\text { Hospital/Nursing home }\end{array}$ & $\begin{array}{c}2314 \\
33\end{array}$ & $\begin{array}{c}320(13.8) \\
7(21.2)\end{array}$ & $\begin{array}{c}1 \\
1.5(08-3.0)\end{array}$ & & \\
\hline $\begin{array}{l}\text { Education of Provider } \\
\text { MBBS } \\
\text { MBBS with Diploma/MD }\end{array}$ & $\begin{array}{c}883 \\
1464 \\
\end{array}$ & $\begin{array}{l}125(14.2) \\
202(13.8)\end{array}$ & $\begin{array}{c}1.0(0.8-1.3) \\
1\end{array}$ & & \\
\hline $\begin{array}{l}\text { District } \\
\text { Kolkota } \\
\text { Howrah } \\
\text { Hooghly } \\
\text { North } 24 \text { Parganas } \\
\text { South 24 Parganas } \\
\text { East Medinipur }\end{array}$ & $\begin{array}{c}1090 \\
434 \\
216 \\
337 \\
181 \\
89\end{array}$ & $\begin{array}{c}175(16.1) \\
54(12.4) \\
22(10.2) \\
39(11.6) \\
34(18.8) \\
3(3.4)\end{array}$ & $\begin{array}{c}4.8(1.6-14.6) \\
3.7(1.2-11.5) \\
3.0(0.9-9.8) \\
3.4(1.1-10.8) \\
4.6(1.8-17.6) \\
1\end{array}$ & $\begin{array}{c}2.8(0.9-8.5) \\
2.5(0.8-8.3) \\
2.0(0.6-6.6) \\
2.3(0.7-7.5) \\
3.3(1.1-10.3) \\
1\end{array}$ & $\begin{array}{l}0.068 \\
0.132 \\
0.245 \\
0.160 \\
0.041\end{array}$ \\
\hline $\begin{array}{l}\text { TB care in the district of residence } \\
\text { Same district } \\
\text { Different district }\end{array}$ & $\begin{array}{l}1982 \\
365\end{array}$ & $\begin{array}{c}279(14.1) \\
48(13.2)\end{array}$ & $1.1(0.8-1.4)$ & & \\
\hline $\begin{array}{l}\text { TB treatment regimen } \\
\text { NTP } \\
\text { Private pharmacy }\end{array}$ & $\begin{array}{c}444 \\
1903\end{array}$ & $\begin{array}{l}156(35.1) \\
171(9.0)\end{array}$ & $\begin{array}{c}3.6(3.0-4.3) \\
1\end{array}$ & $\begin{array}{c}4.0(3.1-5.0) \\
1\end{array}$ & $<0.001$ \\
\hline
\end{tabular}


The NTP can orient the existing field staff or consider a publicprivate mix (PPM) approach to deliver support during treatment for TB patients treated under the private sector. Second, the ICT support through the 'call-centre' and incentives to PPs for ascertainment of TB treatment outcomes might have reduced the rate of 'not evaluated'. The program can try to establish a 'call-centre' with a toll-free number to assist PPs and the TB patients. Third, in the private sector, the majority of TB patients are clinically diagnosed and more than half treated without evaluation for HIV and diabetes mellitus. There is a need to train, support and audit PPs on adherence to standard TB care in India (STCI). Fourth, with high rates of 'unsuccessful outcomes' among those private TB patients receiving free anti-TB drugs from NTP, there is a need to explore the reasons for such findings. Fifth, the program can also advocate for bringing the patients opting for free anti-TB drugs from NTP to the public sector's ambit with directly observed treatment and see whether such a strategy can improve the treatment outcomes.

\section{Conclusions}

The high 'successful outcomes' among TB patients treated by PPs are promising. However, higher 'unsuccessful outcomes' among patients on free NTP drugs need to be explored, and suitable corrective actions have to be taken.

\section{References}

1. WHO. Global tuberculosis report 2019. [cited 2019 Jun 11]. Available from: https://apps.who.int/iris/bitstream/handle/ 10665/329368/9789241565714-eng.pdf

2. Saha I, Paul B. Private sector involvement envisaged in the National Strategic Plan for Tuberculosis Elimination 20172025: Can Tuberculosis Health Action Learning Initiative model act as a road map? Med J Armed Forces India 2019;75:25-7.

3. Suganthi P, Chadha VK, Ahmed J, et al. Health seeking and knowledge about tuberculosis among persons with pulmonary symptoms and tuberculosis cases in Bangalore slums. Int $\mathrm{J}$ Tuberc Lung Dis 2008;12:1268-73.

4. Uplekar M, Juvekar S, Morankar S, et al. Tuberculosis patients and practitioners in private clinics in India. Int J Tuberc Lung Dis 1998;2:324-9.

5. Cazabon D, Alsdurf H, Satyanarayana S, et al. Quality of tuberculosis care in high burden countries: the urgent need to address gaps in the care cascade. Int J Infect Dis 2017;56:111-6.

6. Satyanarayana S, Subbaraman R, Shete P, et al. Quality of tuberculosis care in India: a systematic review. Int $\mathrm{J}$ Tuberc Lung Dis 2015;19:751-63.

7. Das J, Kwan A, Daniels B, et al. Use of standardised patients to assess quality of tuberculosis care: a pilot, cross-sectional study. Lancet Infect Dis 2015;15:1305-13.

8. Bronner Murrison L, Ananthakrishnan R, Sukumar S, et al. How Do urban indian private practitioners diagnose and treat tuberculosis? A cross-sectional study in Chennai. PLoS One 2016;11:e0149862.

9. Ismail I, Bulgiba A. Determinants of unsuccessful tuberculosis treatment outcomes in Malaysian HIV-infected patients. Prev Med 2013;57:S27-30.

10. Kaur G, Goel NK, Kumar D, et al. Treatment outcomes of patients placed on treatment under directly observed therapy short-course (dots). Lung India 2008;25:75-7.

11. Adejumo O, Daniel O, Otesanya A, et al. Evaluation of outcomes of tuberculosis management in private for profit and private-not-for profit directly observed treatment short course facilities in Lagos State, Nigeria. Niger Med J 2017;58:44.

12. Kunoor A, James PT, Raj M, et al. Outcomes of patients treated with individualised anti-tuberculosis regimens in a tertiary care centre in India. Public Heal action 2017;7:308.

13. Fernandez L, Garcia JF, Monroy K, Munoz CA. Treatment outcomes of tuberculosis patients: Cohort 2014 in a private sector in Cali, Colombia. Am J Respir Crit Care Med 2020;201: A5527.

14. Anand T, Babu R, Jacob A, et al. Enhancing the role of private practitioners in tuberculosis prevention and care activities in India. Lung India 2017;34:538.

15. Central TB Division, Directorate General of Health Services. India TB Report 2018. Ministry of Health and Family Welfare [Internet]. 2018. Available from: https://tbcindia.gov.in/showfile.php?lid=3314 